\title{
Elastic phase transitions in solids. High pressure effect
}

\author{
Yu.Kh. Vekilov and O.M. Krasilnikov \\ Department of Theoretical Physics and Quantum Technology, National University of Science and Technology (MISIS) \\ Moscow 119049, Russia \\ E-mail: yuri.vekilov@gmail.com, yuri_vekilov@yahoo.com
}

Received December 12, 2017, published online April 25, 2018

\begin{abstract}
At high pressures (the pressure is comparable with the bulk modulus) the crystalline lattice may become unstable relative to the uniform shear deformations, and in a result the low symmetric crystalline structures will appear (the so-called "elastic phase transitions"). The order parameters at these transitions are the components of the finite deformations tensor. The stability of the high-pressure phases is defined by the nonlinear elasticity of the lattice (the third, fourth etc. order elastic constants). Here the different cases of the stability loss at hydrostatic pressure for the cubic structures are considered. The relation between the second, third and fourth order elastic constants is given, which defines the possibility of the first order deformation phase transition. The jump of the order parameter and the height of the potential barrier are defined by the third and fourth order elastic constants. As an example, the experimentally observed elastic phase transition in vanadium at $P \approx 69 \mathrm{GPa}$ from bcc to the rhombohedral phase is analyzed, and the possible structural transitions in bcc Mo and $\mathrm{W}$ at $P \geq 700 \mathrm{GPa}$ are also considered.
\end{abstract}

PACS: 61.50.Ks Crystallographic aspects of phase transformations; pressure effects;

61.66.Bi Elemental solids;

62.20.D- Elasticity, elastic constants;

62.50.-p High-pressure effects in solids and liquids.

Keywords: phase transitions, high pressures, nonlinear elastic properties.

\section{Introduction}

The elastic deformations accompany nearly all phase transitions in solids. In the most cases the deformation is not the order parameter, but is connected with it by the striction interaction. In the considered structural transformations, the order parameter is the uniform deformation. These transformations were called "the elastic phase transitions". At these transitions the number of atoms in the unit cell is conserved, the symmetry point group of a new phase is subgroup of the point group of the initial phase, the translation symmetry is not changed [1].

The elastic phase transitions can be observed with the temperature $T$ and pressure $P$ changes in a result, of the stability loss of the crystalline lattice relative to the shear deformations. As a result, the transition takes place in the spontaneously deformed state with the lower symmetry. It was shown [2,3], that the critical phenomena at the elastic phase transitions are strongly suppressed, and this gives the possibility to use the Landau theory of the phase transitions [4], where the order parameters are the components of the finite deformations tensor, because at these transitions the lattice deformations are although small but finite.
The example of these transitions with the temperature changes are the structural transitions in $\mathrm{Nb}_{3} \mathrm{Sn}$ and $\mathrm{V}_{3} \mathrm{Si}$. In these compounds the transitions from cubical phase to the tetragonal are observed at temperatures slightly exceeded the superconductivity transition temperatures: in $\mathrm{Nb}_{3} \mathrm{Sn}$ at $T=43 \mathrm{~K}$, in $\mathrm{V}_{3} \mathrm{Si}$ at $T=21 \mathrm{~K}$. In both cases the structural transitions are accompanied by the strong softening (practically to zero) of the elastic constant $C^{\prime}=\left(C_{11}-C_{12}\right) / 2$, corresponding to the tetragonal deformation, and are close to the second order phase transition [5].

The elastic phase transition recently was observed in vanadium at $P \approx 69 \mathrm{GPa}$ (the room temperature) [6]. The experimental investigation of the vanadium structure has shown that the bcc phase of vanadium at this pressure transforms in the rhombohedral structure. Wherein the volume abrupt change is absent, and the second order phase transition should take place [6]. This behavior obviously is connected with the strong softening of the shear effective elastic constant $\tilde{C}_{44}$, which was observed in the DFT calculations [7-9]. The feature of these transitions is that the elastic phase transitions are possible when the pressures are comparable with the material bulk modulus (as a rule, the tens and hundreds GPa). There is the growing 
interest to this problem $[9,10]$, because the using of the cells with diamond anvils gives possibility to do experiments at the pressures 500-600 GPa [11], and the structural investigations based on the synchrotron $x$-ray irradiation give the possibility to investigate the high pressure phases in the real time regime. In the present work the peculiarities of the elastic phase transitions under pressure are considered in the framework of the Landau theory, the quantitative estimations are performed for the transition metals with cubical lattice: vanadium, molybdenum and tungsten.

\section{The expansion of the thermodynamic potential}

The thermodynamic potential at the pressure $P$ and temperature $T$ is the Gibbs free energy $G$. For taking into account the nonlinear effects it is need to choose as the order parameters the components of the Lagrange finite deformations tensor $\eta_{i j}$ [12]. Following to [13], we expand $G$ near the equilibrium state at given $P$ and $T$ over the components $\eta_{i j}$.

Let us the spontaneous deformations $\eta_{i j}$ are isothermal. Then, the change $\Delta G=G(P, T, \eta)-G(P, T, 0)$ on the unit volume in the initial state $\left(V_{0}\right)$ is

$$
\begin{aligned}
\frac{\Delta G}{V_{0}} & =\frac{1}{2} \tilde{C}_{i j k l} \eta_{i j} \eta_{k l}+\frac{1}{6} \tilde{C}_{i j k l m n} \eta_{i j} \eta_{k l} \eta_{m n}+ \\
& +\frac{1}{24} \tilde{C}_{i j k l m n p q} \eta_{i j} \eta_{k l} \eta_{m n} \eta_{p q}+\ldots
\end{aligned}
$$

Here and below the summation over the repeated indexes from 1 to 3 takes place, the linear contribution is absent, the fourth order contribution is included. Here

$$
\tilde{C}_{i j k l \ldots .}=\frac{1}{V_{0}}\left(\frac{\partial^{n} G}{\partial \eta_{i j} \partial \eta_{k l} \cdot .}\right)_{0}
$$

are $n$-order ( $n=2,3,4)$ the isothermal effective elastic constants at the given load and temperature $T$, which characterize the elastic properties of a loaded crystal [14]. The elastic constants $\tilde{C}_{i j k l . .}$ in the absence of a load are coincide with the usual Brugger elastic constants [15]. With $\tilde{C}_{i j k l . .}$ the all formula of the elasticity theory are same as for the loaded, as for the unloaded crystals. In particular, the stability conditions at uniform deformations for the loaded crystal are coincide with the well-known Born conditions.
At the given $P$ and $T$ the system state will be stable, if the quadratic form in (1) will be positively defined. As a result, for the cubic crystal we have the following stability conditions relative to the uniform compression and shear deformations

$$
\begin{gathered}
\tilde{C}_{11}+2 \tilde{C}_{12}>0, \\
\tilde{C}_{11}-\tilde{C}_{12}>0, \\
\tilde{C}_{44}>0 .
\end{gathered}
$$

Here $\tilde{C}_{\alpha \beta}$ are the effective second order elastic constants in Voigt notations: $\alpha, \beta$ are from 1 to 6 according to the rule $11 \rightarrow 1,22 \rightarrow 2$, 33 $\rightarrow 3,23 \rightarrow 4,13 \rightarrow 5,12 \rightarrow 6$.

With the pressure and temperature changes the conditions (3) can be destroyed, and as a consequence, the structural transition in the spontaneously deformed state takes place, which stability is defined by the nonlinear elasticity of the material: by the anharmonic contributions of the third, fourth and higher order. So, the value of the spontaneous deformation, which characterize the new equilibrium structure, is defined by the higher order nonlinear contributions in the Gibbs energy. The conditions (3a) for the expansion-contraction can be destroyed, as the calculations show, only at the negative pressures [16]. The spinodal instability $P<0$, i.e., the uniform expansion of the lattice can be realized in the shock wave experiments [17], or due to doping of the lattice by the higher size atoms [18]. Due to this, we will discuss the conditions (3b) and (3c), which define the crystalline lattice stability relative to the shear deformations. The decomposition (1) for the cubic lattice can be created using the results of the papers $[19,20]$, where all nonzero the second-fourth order elastic constants for each 32 symmetry point group are given.

In Table 1 the different kinds of the spontaneous deformation are shown, corresponding to the stability loss of crystals with the cubical structures. In the considered cases the decomposition of the thermodynamic potential (1) is following:

$$
\frac{\Delta G(P, \eta, T)}{V_{0}}=\frac{1}{2} q \eta^{2}+\frac{1}{3} v \eta^{3}+\frac{1}{4} w \eta^{4}+\ldots .
$$

Table 1. The elastic phase transitions under pressure in the cubical crystals

\begin{tabular}{c|c|c|c|c|c}
\hline \hline No. & $\begin{array}{c}\text { Spontaneous } \\
\text { deformation }\end{array}$ & $q$ & $v$ & $w$ & $\begin{array}{c}\text { Symmetry changes } \\
\text { at the transition }\end{array}$ \\
\hline \hline 1 & $-\eta_{11}=-\eta_{22}=0.5 \eta_{33}=\eta$ & $6\left(\tilde{C}_{11}-\tilde{C}_{12}\right)$ & $3\left(\tilde{C}_{111}-3 \tilde{C}_{112}+2 \tilde{C}_{123}\right)$ & $3\left(\tilde{C}_{1111}-4 \tilde{C}_{1112}+3 \tilde{C}_{1122}\right)$ & $\Gamma_{c} \rightarrow \Gamma_{q}$ \\
$\Gamma_{C}^{f}, \Gamma_{c}^{v} \rightarrow \Gamma_{q}^{v}$ \\
\hline 2
\end{tabular}

Comments: * - due to the deformation symmetry. 
Here the coefficients $q, v$ and $w$ for each case are given in Table 1 , where $\tilde{C}_{\alpha \beta . .}$ are the isothermal effective elastic constants at the pressure $P$ in Voigt notations. Here also the different variants of structural transformations for bcc and fcc lattices due to the stability loss relative to shear deformations are given, obtained by the theoretical-group analysis of the Brave lattices symmetry [21]. If the third power contribution in (4) is zero (the case 2), then the second order phase transition can take place.

Now consider the Eq. (4) when the third order contribution is not zero (the cases 1,3 ). The elastic constants calculations confirm that from the point of the stability loss $q$ and $w$ are positive, but $v$ is less of zero. The Eq. (4) contains the three parameters, and each of them depends on $P$ and $T$. Dividing the both parts of this equation on $G_{0}=v^{4} / w^{3}$ we get the dimensionless equation with one coefficient [22]

$$
f(a, x)=a \frac{x^{2}}{2}-\frac{x^{3}}{3}+\frac{x^{4}}{4},
$$

where

$$
f(a, x)=\frac{\Delta G}{V_{0} G_{0}}, \quad x=\frac{w}{|v|} \eta, \quad a=\frac{q w}{v^{2}} .
$$

The coefficient $a$ takes into account the changes with the pressure and temperature of the second, third and fourth order elastic constants. Depending of it the Eq. (5) has the different maxima and minima (see Fig. 1). Their position is defined by the condition $\partial f / \partial x=0$. In a result, we'll get the equation $a x-x^{2}+x^{3}=0$. The roots of it are $x=0$ and $x=(1 \pm \sqrt{1-4 a}) / 2$. It follows from this, that if $a>1 / 4$, $f(a, x)$ has only one minimum at $x=0$ the appropriate initial phase. If $a=1 / 4$, then only one minimum at $x=0$ and the inflection point at $x=1 / 2$ exist (see Fig. 1, curve 1 ). When $2 / 9<a<2 / 8(1 / 4)$, the curve $f(a, x)$ has the two minima: the one is stable at $x=0$, the second is metastable at $x=(1+\sqrt{1-4 a}) / 2$, and relative maximum at $x=(1-\sqrt{1-4 a}) / 2$ (Fig. 1, curve 2).



Fig. 1. The dimensionless Gibbs potential (5) at different coefficient a values: $a=1 / 4$ (1), $a=2.1 / 9$ (2), $a=2 / 9$ (3), $a=1.4 / 9$ (4).
The value of the coefficient $a$ for the first order transition we obtain from the solution of the system equations $f(a, x)=0$ and $\partial f / \partial x=0$. In a result, we get $a=2 / 9$. At this value of the coefficient $a$ the curve (5) has the two minima for $f(a, x)=0$, at $x=0$ and $x=2 / 3$, and maximum at $x=1 / 3$ (see Fig. 1, curve 3). The potential barrier height and the jump of the order parameter can be obtained from the relations (5) and (6). In a result,

$$
\frac{\Delta G}{V_{0}}=\frac{1}{324} \frac{v^{4}}{w^{3}} ; \quad \eta=\frac{2|v|}{3 w} .
$$

In the expressions needed for the calculation of $q, v$ and $w$ in all considered cases are given in Table 1. Thus, the potential barrier height and the jump of the order parameter are defined by relations between the third and fourth order elastic constants, i.e., by the nonlinear elasticity of crystalline lattice. It is seen from the expression for the parameter $a$ (see (6)) that, when the value of $v$ is in proximity to $w$ (the third order anharmonic contribution is nearly equal to fourth order contribution), then the first order phase transition can be realized at the rather high values of $q$. At this transition the unit cell volume is changed in the second order on $\eta$, because [16] $\Delta V / V_{0}=-2\left(\eta_{12}^{2}+\eta_{13}^{2}+\eta_{23}^{2}\right)+$ $+8 \eta_{12} \eta_{13} \eta_{23}$. Thus, the structural distortion takes place with small (in the second order over deformation), but the negative volume change. If $0<a<2 / 9, f(a, x)$ then besides the metastable minimum at $x=0$, the stable minimum exists at $x>2 / 3$ with maximum $f(a, x)>0$ for $x$ in the interval between of these minima (Fig. 1, curve 4 ). The first order phase transition (closed to the second order) takes place, because the potential barrier height decreases with the diminishing of $a$. The interval of the $a$ values, where the first order transition is possible is $0<q w \leq(2 / 9) v^{2}$.

Thus, for the analysis of the elastic phase transitions it is need to have the data for the second-fourth order elastic constants at the different pressures. The experimental data for the different order elastic constants at high pressures are practically absent, and it is due to the big problems of the sound velocity measurements in the megabar pressure interval for the different crystallographic directions, the second harmonic generation, or the Raman scattering of light, which are usually used for the definition of elastic constants. It is possible to calculate the second and the higher order elastic constants at the different pressures from the "first principles" in the framework of the density functional theory. Here we shortly consider the example of such calculation following to our earlier work [14].

\section{The $n$-order effective elastic constants at the hydrostatic pressure}

The density functional method gives the possibility to calculate the full energy of a crystal at any atomic volume (i.e., at any pressure). So we express $\Delta G / V_{0}$ via the change 
of the Helmholtz free energy $\Delta F$. We obtain at hydrostatic compression $P$

$$
\frac{\Delta G}{V_{0}}=\frac{\Delta F}{V_{0}}+P \frac{\Delta V}{V_{0}}
$$

Where $\Delta V=V-V_{0}$ is the volume change due to deformation, defined by the tensor components $\eta_{i j}$. It is possible to present $\Delta F / V_{0}[12]$ as

$$
\begin{aligned}
\frac{\Delta F}{V_{0}}=-P \eta_{i i} & +\frac{1}{2} C_{i j k l} \eta_{i j} \eta_{k l}+\frac{1}{6} C_{i j k l m n} \eta_{i j} \eta_{k l} \eta_{m n}+ \\
+ & \frac{1}{24} C_{i j k l m n p q} \eta_{i j} \eta_{k l} \eta_{m n} \eta_{p q} .
\end{aligned}
$$

Here

$$
C_{i j k l . . .}=\frac{1}{V_{0}}\left(\frac{\partial^{n} F}{\partial \eta_{i j} \partial \eta_{k l} . \cdot}\right)_{0}
$$

The constants $C_{i j k l . . .}$ are the generalization of the $n$-order elastic constants introduced by Brugger [15] for the loaded state. They are not fully define the elastic properties of a loaded crystal [23], for them the Cauchy relations are fulfilled, in contrast to $\tilde{C}_{i j k l . . . .}$.

Now we express the $n$-order effective elastic constants $\tilde{C}_{i j k l \ldots .}$ at the hydrostatic loading via the Brugger type $C_{i j k l . . .}$ and the pressure $P$. We take in to account that $\Delta V / V_{0}=J-1$, where $J=\operatorname{det}\left|\alpha_{i j}\right|$ [12]. Here $\alpha_{k i}=\partial r_{k} / \partial R_{i}$ is the deformation gradient, $r_{k}$ and $R_{i}$ are the Cartesian coordinates of the point in a crystal in the deformed and the initial states. Then we express $\alpha_{i j}$ via $\eta_{i j}$ to get the relations between $\tilde{C}_{i j k l . . .}$ and $C_{i j k l . .}$ :

$$
\eta_{i j}=\frac{1}{2}\left(\alpha_{k i} \alpha_{k j}-\delta_{i j}\right)
$$

where $\delta_{i j}$ is the Kronecker symbol. Then, $\alpha_{i j}$ can be expressed via $u_{i j}=\partial u_{i} / \partial R_{j}\left(u_{i}=r_{i}-R_{i}\right)$ :

$$
\alpha_{i j}=\delta_{i j}+u_{i j}
$$

In a result, $\eta_{i j}=\frac{1}{2}\left(u_{i j}+u_{j i}+u_{k i} u_{k j}\right)$. Then we'll consider the "pure" deformations (the rotation of a crystal is absent, $u_{i j}=u_{j i}$ ), and so

$$
\eta_{i j}=u_{i j}+\frac{1}{2} u_{k i} u_{k j}
$$

The inversion of (11), using (12) and (13) (the fourth order contributions are taken in to account), gives

$\alpha_{i j}=\delta_{i j}+\eta_{i j}-\frac{1}{2} \eta_{k i} \eta_{k j}+\frac{1}{2} \eta_{r k} \eta_{r i} \eta_{k j}-\frac{5}{8} \eta_{k j} \eta_{m k} \eta_{m n} \eta_{n i}$.

Using the relations (2), (8), (10) and (14) we'll find the relations between $\tilde{C}_{i j k l \ldots . . .}$ and $C_{i j k l . . . .}$. The results are given in Table 2 (the 2, 3 and 4 order elastic constants are given in Voigt notations).

In a result, for the calculation of any order $\tilde{C}_{\alpha \beta}$.. it is possible to use the decomposition (9). The linear contribution defines the pressure, the higher order contributions define the elastic constants $C_{\alpha \beta}$. (the Brugger type elastic constants). Then, using the relations given in Table 2 the effective elastic constants $\tilde{C}_{\alpha \beta}$.. of the necessary order are found.

\section{Structural transformations in vanadium at high pressure}

The phase transition from bcc to rhombohedral phase was observed at room temperature in vanadium with no volume changes [6]. As the "first principles" calculations shown, in vanadium the elastic constant $\tilde{C}_{44}$, corresponding to the rhombohedral deformation, becomes negative [7-9]. Consider the results of the second-fourth order constants calculations at the different pressures $(T=0 \mathrm{~K})$ from the paper [9] to analyze the structural transition bcc-rhombohedral phase. The required constants are given in Table 1, line 3. Using them, we can obtain the value of the coefficient $a$ (see (5) and (6) at the different pressures) in the vicinity of the phase transition point (50-70 GPa). In a result, we get: $P=55 \mathrm{GPa}, a=3.9 ; P=63 \mathrm{GPa}, a=1.4$; $P=68 \mathrm{GPa}, a=0.21$. So, at $P \approx 68 \mathrm{GPa}$ the value of the coefficient $a$ becomes equal to the critical value $a=2 / 9$, and bcc structure $\left(\Gamma_{c}^{v}\right)$ can be transformed in to rhombohedral phase $\left(\Gamma_{\mathrm{rh}}\right)$ (see Table 1 , line 3 ). The jump of the order parameter at this transition we'll obtain from the relation (7): $\eta_{12}=\eta_{13}=\eta_{23}=\eta / 2=(1 / 3)|v| / w=0.0075$. The small value of the order parameter shows that it is the first order transition close to the second order. The results agree with the experimental data [6].

In the papers $[9,24]$ the results of the phonon dispersion calculations in vanadium at the different pressures by DFT

Table 2. The relations between $\tilde{C}_{\alpha \beta . .}$ and $C_{\alpha \beta}$..

\begin{tabular}{cccc}
\hline \hline$\tilde{C}_{\alpha \beta}$ & $\tilde{C}_{\alpha \beta \gamma}$ & $\tilde{C}_{\alpha \beta \gamma \delta}$ & \\
\hline \hline$\tilde{C}_{11}=C_{11}-P$ & $\tilde{C}_{111}=C_{111}+3 P$ & $\tilde{C}_{1111}=C_{1111}-15 P$ & $\tilde{C}_{4444}=C_{4444}-3 P$ \\
$\tilde{C}_{12}=C_{12}+P$ & $\tilde{C}_{112}=C_{112}-P$ & $\tilde{C}_{1112}=C_{1112}+3 P$ & $\tilde{C}_{4455}=C_{4455}-P$ \\
$\tilde{C}_{44}=C_{44}-P$ & $\tilde{C}_{123}=C_{123}+P$ & $\tilde{C}_{1122}=C_{1122}+P$ & \\
& $\tilde{C}_{456}=C_{456}+P$ & & \\
\hline \hline
\end{tabular}


perturbation method are given. These results show, that the both transverse modes (directions $\Gamma-\mathrm{H}$ and $\Gamma-\mathrm{N}$ ), connected with the elastic constant $\tilde{C}_{44}$, suffer the strong softening in the vicinity of the $\mathrm{BZ}$ center ( $\Gamma$ point). at the pressures 70-75 GPa. With the pressure increasing the tilt of both branches continues to decrease. At the same time the second transverse branch practically is not changed in this pressure interval. So, the pressure dependence of the dispersion curves agree with the elastic constants data.

\section{The softening of the effective elastic constant $\tilde{C}^{\prime}$ in bcc molybdenum at high pressures}

The effective elastic constants and the phonon dispersion curves for molybdenum in the pressure interval 0-1400 GPa ( $T=0 \mathrm{~K}$ ) were obtained in work [25] and are shown on Figs. 2, 3. It is seen on Fig. 2, that $\tilde{C}^{\prime}$ at $P>500 \mathrm{GPa}$ suffers the strong softening (what is the precursor of the phase transition) and goes to zero at $P \approx 1400 \mathrm{GPa}\left(V / V_{0} \approx 0.42\right)$. The bcc lattice can suffer the elastic phase transition at these pressures in to the tetragonal or orthorhombic structures (the stability condition (3b) is destroyed). However, according to [26-28] the bcc structure of molybdenum at $P \approx \approx 700 \mathrm{GPa}(T=0 \mathrm{~K})$ becomes thermodynamically unstable and should transfer in fcc or hexagonal phase. The estimations of $a$ (formula (5)) show that at these pressures its value essentially exceeds the critical value $2 / 9$, when the first order elastic phase transition can take place. The calculations of the stability criteria of the bcc, fcc and hcp structures of molybdenum show [25], that at the atmospheric pressure the both structures are unstable relative to the shear deformations $\left(\tilde{C}_{44}<0\right.$ and $\left.\tilde{C}^{\prime}<0\right)$. They become stable only at the high pressures: hcp at $P \geq 150 \mathrm{GPa}$, bcc at $P \geq 300 \mathrm{GPa}$, and so the hexagonal structure at the phase transition is more preferable.

The calculated dispersion laws for bcc molybdenum in the pressure interval 900-1050 GPa are given on Fig. 3. It

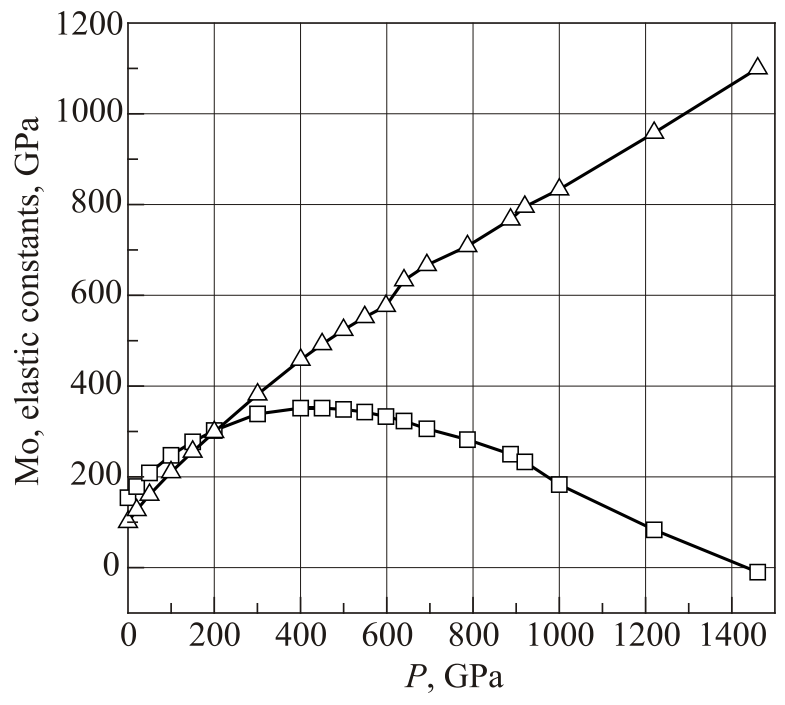

Fig. 2. The pressure dependence of the shear elastic constants of bcc Mo $\tilde{C}^{\prime}=\left(\tilde{C}_{11}-\tilde{C}_{12}\right) / 2(\square), \tilde{C}_{44}(\triangle)$.

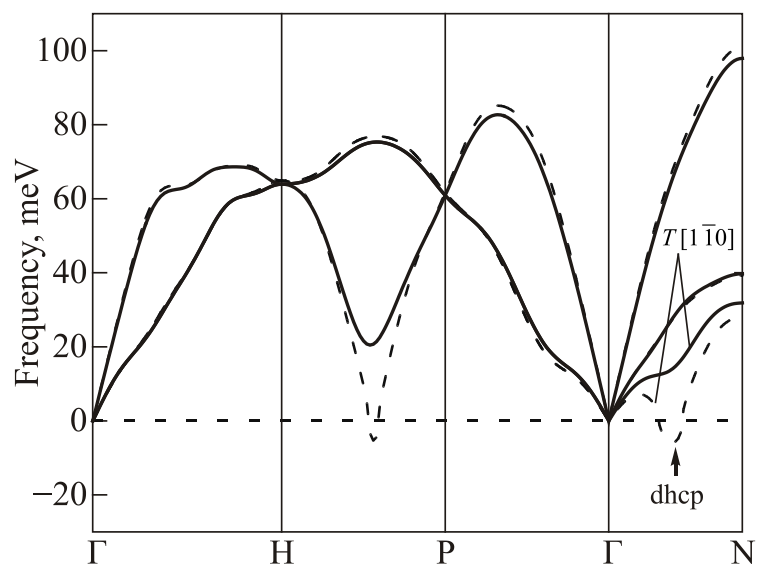

Fig. 3. The dispersion laws for bcc-Mo at the $900 \mathrm{GPa}$ pressure (solid line) and $1050 \mathrm{GPa}$ (dotted line). The negative values are for the imaginary frequencies.

is seen that the branch of the transverse modes $T_{[1 \overline{110}]}[\zeta \zeta 0]$, connected with the elastic constant $\tilde{C}^{\prime}$ (the low curve in the direction $\Gamma-N$ ), near the $\Gamma$ point experiences at $900 \mathrm{GPa}$ the strong softening. Besides, the strong softening is observed also for the longitudinal branch $L[\zeta \zeta \zeta]$ in the direction $H-P$. With the further increase of pressure ( $P=1050 \mathrm{GPa})$ the frequencies of these branches near the frequency modes $T_{[1 \overline{110}]}\left[\frac{1}{4} \frac{1}{4} 0\right]$ and $L\left[\frac{2}{3} \frac{2}{3} \frac{2}{3}\right]$ become image. It is shown in the paper [18], that the instability of the bcc lattice relative to the vibration mode $T_{[\overline{110}]}\left[\frac{1}{4} \frac{1}{4} 0\right]$ with the softening of the shear elastic constants $\tilde{C}^{\prime}$ can induce the transition between bcc and double hcp structures. The small value of $\tilde{C}^{\prime}$ creates the small energetic barrier for this structural transformation. This will be the first order transition, and it occurs before the phonon mode in the initial lattice will become unstable. So, the structural transition in molybdenum in the double hcp should occur before the $\tilde{C}^{\prime}$ constant will become zero.

\section{Tungsten, the pressure effect}

The second-fourth order elastic constants of bcc W in the pressure interval $0-600 \mathrm{GPa}(T=0 \mathrm{~K})$ are given in our work [29]. The pressure dependence of the elastic constants $\tilde{C}^{\prime}$ and $\tilde{C}_{44}$ is shown on Fig. 4. It is seen, that as in molybdenum $\tilde{C}^{\prime}$ is increasing with the pressure much lower then $\tilde{C}_{44}$, and at $P \approx 600 \mathrm{GPa}$ the tendency to softening is observed. The same tendency is observed in the calculation results of the corresponding phonon modes. We have calculated the Grüneisen parameters $\gamma_{j}$ for the bcc $\mathrm{W}$ in the pressure interval 0-600 GPa for the three long-wave acoustic modes in the direction [110], using the data for the second and third order elastic constants. 


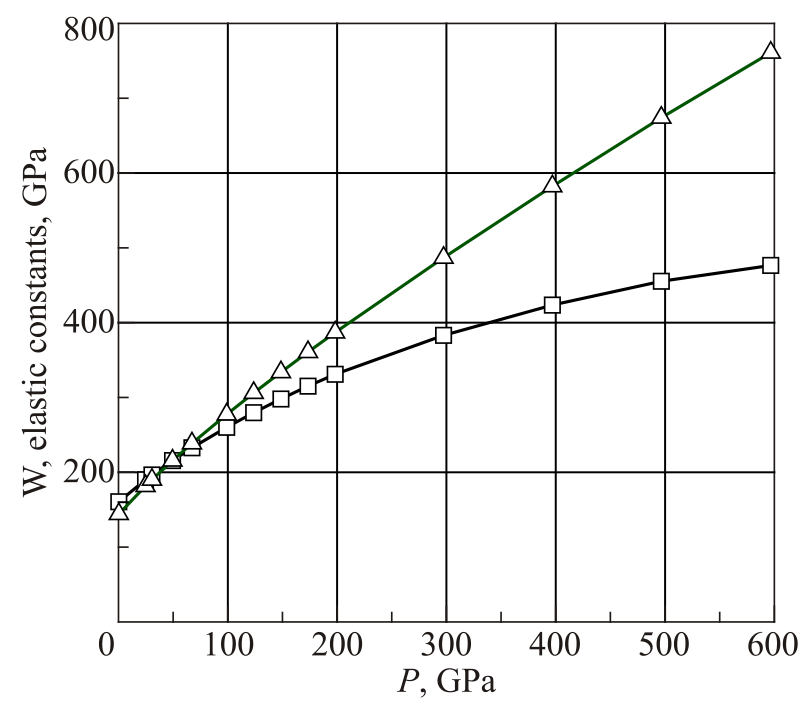

Fig. 4. The pressure dependence of the shear elastic constants for the bcc W. $\tilde{C}^{\prime}=\left(\tilde{C}_{11}-\tilde{C}_{12}\right) / 2(\square), \tilde{C}_{44}(\triangle)$.

The constants $\gamma_{j}$ are defined by the relation $\gamma_{j}=-\left(V_{0} / \omega_{j}\right)\left(d \omega_{j} / d V\right)$, where $\omega_{j}$ is the frequency of the $j$ th normal mode of the lattice vibrations. The relations between $\gamma_{j}$ and $\tilde{C}_{\alpha \beta \gamma}$ are taken from [30, p. 120]. The values of $\gamma_{j}$ for the one longitude and the two transverse longwave modes in direction [110] are given on Fig. 5. It is seen that the Grüneisen parameters for the longitudinal and for the one transverse (the polarization [001] long-wave acoustic modes are nearly unchanged with the pressure increasing. At the same time $\gamma_{j}$ of the transverse mode with the polarization [110] is decreased with the pressure increasing nearly by linear law and goes over zero at $P \approx 600 \mathrm{GPa}$. This points out, that at the pressures $P>600 \mathrm{GPa}$ the vibration frequencies (the elastic constant $\left.\tilde{C}^{\prime}\right)$ becomes to decrease $\left(\gamma_{j}\right.$ is negative). In a result, the bcc structure of $\mathrm{W}$ can be unstable to the tetragonal

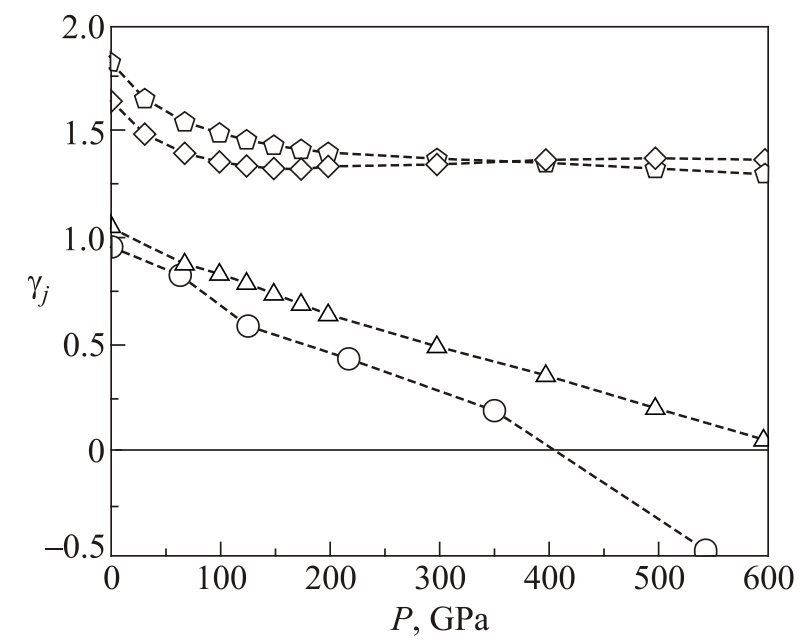

Fig. 5. Pressure dependence of Grüneisen parameters $\gamma_{j}$ for bcc tungsten and molybdenum. $L_{[110]}[110]$ mode $(\triangle), T_{[001]}[110]$ mode $(\diamond), T_{[1 \overline{1} 0]}[110]$ mode $(\triangle)$ of $W$ and $T_{[1 \overline{1} 0]}[110]$ mode $(\bigcirc)$ of Mo. deformation in the pressure megabar diapason. For comparison, on the Fig. 5 the pressure dependence of $\gamma_{j}$ for the same mode bcc Mo is given. It is seen that $\gamma_{j}$ is also diminished with the pressure increasing and becomes negative at $P \geq 400 \mathrm{GPa}$. This result is in agreement with the data on the Fig. 2: the elastic constant $\tilde{C}^{\prime}$ of Mo becomes to diminish at $P \geq 400 \mathrm{GPa}$.

The results of the phonon dispersion laws for bcc $\mathrm{W}$ at the high pressures are given in [18]. The frequencies of the long-wave transverse vibrations in the direction [110] with the polarization [110] show the strong softening at $P \geq$ $\geq 1000 \mathrm{GPa}$, and at the same time, the frequencies of $T_{[110]}\left[\frac{1}{4} \frac{1}{4} 0\right]$ shear mode tend to zero. As it was show in [18], such instability together with $\tilde{C}^{\prime}$ softening leads to bcc $\rightarrow$ dhcp transition.

\section{Conclusion}

The peculiarities of elastic phase transitions in crystals at high pressures are analyzed in the framework of the Landau theory of the phase transitions. The definition of the $n$-order $(n \geq 2)$ effective elastic constants for a loaded crystal is given. The method of their calculations from the "first principles" at the hydrostatic pressure is developed. The stability criteria to the shear deformations at the pressure $P$ are expressed via the effective second order elastic constants. The different cases of the stability loss relative to the shear deformations at the given $P$ are considered, and the Brave lattices of the high-pressure phases, obtained in a result of the elastic phase transitions are defined. The stability of these phases is defined by the nonlinear elasticity of the lattice (by the third and fourth order elastic constants). The relation between the second, third and fourth order elastic constants is obtained, at which the first order transition is possible. The jump of the order parameter and the height of the potential barrier at the transition are defined by the third and fourth order elastic constants. As an example, the experimentally observed phase transition in vanadium from bcc to the rhombohedral phase at $P \approx 69 \mathrm{GPa}$ is analyzed in framework of the developed method. The possibility of the structural transitions in the bcc Mo and $\mathrm{W}$ at high pressures is also considered. The necessary values of the different order elastic constants for $\mathrm{V}$, Mo and $\mathrm{W}$ are calculated in the framework of the density functional theory.

In conclusion, the investigation of the matter at high pressures is the important part of the scientific heritage of A.A. Abrikosov. He was the head of the Institute of High Pressure Physics RAN and was the first, who predicted the transformation of hydrogen in the metallic phase at several megabar pressure (see his papers in 1954-1963). The convincing proofs of this transition are experimentally obtained in the present time (R.P. Dias and I.F. Silvera, Science 26 Jan. 2017). 


\section{Acknowledgment}

The work is executed at financial support of the Ministry of Education and Science of the Russian Federation (Grant No. 14.Y26.31.0005) and the Russian Foundation for Basic Research (Grant 16-02-00699 and Grant 16-0201027).

1. V.L. Indenbom, Kristallogr. 5, 115 (1960) [Sov. Phys. Crystalogr. 5, 106 (1960)].

2. D.E. Khmel'nitskii, Sov. Phys. Solid State 16, 2079 (1975); Fiz. Tverd. Tela 16, 3188 (1974).

3. R.A. Cowley, Phys. Rev. B 13, 4877 (1976).

4. L.D. Landau and E.M. Lifshitz, Statistical Physics, Pegramon Press, Oxford (1980), vol. 1.

5. S.V. Vonsovskii, Yu.A. Izyumov, and E.Z. Kurmayev, Superconductivity of Transition Metals, their Alloys and Compounds, Nauka, Moscow (1977) (in Russian).

6. Y. Ding, R. Ahuja, J. Shu, P. Chow, W. Luo, and K. Mao, Phys. Rev. Lett. 98, 085502 (2007).

7. A. Landa, J. Klepeis, P. Soderlind, I. Naumov, O. Velikokhatny, L.Vitos, and A. Ruban, J. Phys.: Condens. Matter 18, 5079 (2006).

8. L. Koci, Y. Ma, A. Oganov, P. Souvatzis, and R. Ahuja, Phys. Rev. B 77, 214101 (2008).

9. O.M. Krasilnikov, Yu.Kh. Vekilov, I.Yu. Mosygin, E.I. Isaev, and N.G. Bondarenko, J. Phys.: Condens. Matter 24, 195402 (2012).

10. Y.X. Wang, Q. Wu, X.R. Chen, and H.Y. Geng, Sci. Rep. 6, 32419 (2016).

11. L. Dubrovinsky, N. Dubrovinskaja, and V.B. Prakapenka, Nat. Commun. 3,1163 (2012).

12. D.C. Wallace, in: Solid State Physics, H. Ehrenreich, F. Seitz, and D. Turnbull (eds.), Academic Press, New York (1970), vol. 25, p. 301.

13. Yu.Kh. Vekilov, O.M. Krasilnikov, M.P. Belov, and A.V. Lugovskoy, Phys. Usp. 57, 897 (2014).
14. O.M. Krasil'nikov, Yu.Kh. Vekilov, and I.Yu. Mosyagin, Zh. Eksp. Teor. Fiz. 142, 266 (2012) [JETP 115, 237 (2012)].

15. K. Brugger, Phys. Rev. 133, A1611 (1964).

16. O.M. Krasil'nikov, Yu.Kh. Vekilov, E.I. Isaev, and N.G. Bondarenko, Zh. Eksp. Teor. Fiz. 139, 281 (2011) [JETP 112, 240 (2011)].

17. G.V. Sin'ko and N.A. Smirnov, Pis'ma Zh. Eksp. Teor. Fiz. 7, 217 (2002) [JETP Lett. 75, 184 (2002)].

18. G. Grimvall, B. Magyari-Kope, V. Ozolins, and K.A. Persson, Rev. Mod. Phys. 84, 945 (2012).

19. H.B. Huntington, Solid State Phys. 7, 213 (1958); Usp. Fiz. Nauk 74, 303 (1961).

20. D.Y. Chung and and Y. Li, Acta Cryst. A 30, 1 (1974).

21. G.L. Bir and G.E. Pikus, Symmetry and Strain-Induced Effects in Semiconductors, Wiley, New York (1974).

22. J.A. Krumhans and R.J. Gooding, Phys. Rev. B 35, 304 (1989).

23. J.W. Martin, Phys. C: Solid State Phys. 8, 2837 (1975).

24. W. Luo, R. Ahuja, Y. Ding, and H.-K. Mao, Proc. Natl. Acad. Sci. 104, 16428 (2007).

25. O.M. Krasilnikov, M.P. Belov, A.V. Lugovskoy, I.Yu. Mosyagin, and Yu.Kh. Vekilov, Comp. Matter Sci. 81, 313 (2014).

26. A.B. Belonoshko, S.I. Simak, A.E. Kochetov, B. Johanson, L. Burakovsky, and D.I. Preston, Phys. Rev. Lett. 92, 195701 (2004).

27. A.B. Belonoshko, L. Burakovsky, S.P. Chen, B. Johanson, A.S. Mikhaylushkin, D.I. Preston, S.I. Simak, and D.C. Swift, Phys. Rev. Lett. 100, 135701 (2008).

28. A.S. Mikhaylushkin, S.I. Simak, L. Burakovsky, S.P. Chen, B. Johanson, D.I. Preston, D.C. Swift, and A.B. Belonoshko, Phys. Rev. Lett. 101, 049602 (2008).

29. Yu.Kh. Vekilov, O.M. Krasilnikov, A.V. Lugovskoy, and Yu.E. Lozovik, Phys. Rev. B 94, 104114 (2016).

30. H. Ledbetter and S. Kim, in: Elastic Grüneisen Parameters of Cubic Elements and Compounds: Handbook of Elastic Properties of Solids, Liquids, and Gasses, Academic Press, (2001), vol. 2, p. 107. 